\title{
In-situ Observation of Ionospheric Plasma Aboard ESEO
}

\author{
Viktor Qiao, Tamás Kőnig, Zsolt Váradi, \\ József Szabó, Antal Bánfalvi \\ Department of Broadband Infocommunications and \\ Electromagnetic Theory \\ Budapest University of Technology and Economics \\ Budapest, Hungary
}

\author{
Árpád Kis \\ Geodetic and Geophysical Institute \\ Research Centre for Astronomy and Earth Sciences, \\ HAS Sopron, \\ Hungary
}

\begin{abstract}
This paper presents the expected scientific results of the Langmuir Probe Experiment (LMP) aboard the European Student Earth Orbiter (ESEO). LMP is an instrument designed to study the plasma environment in Earth's ionosphere. The instrument was developed by the Laboratory of Space Technology at Budapest University of Technology and Economics and Geodetic and Geophysical Institute of the Hungarian Academy of Sciences.
\end{abstract}

Keywords-ESEO, micro-satellite, Earth observation, Langmuir probe, plasma, ionosphere

\section{ABOUT ESEO}

The official description of the mission is as follows:

'ESEO is an ESA micro-satellite project with an educational objective: for the participating university students to acquire hands-on experience of a real space project, in order to prepare a well-qualified technical workforce for the European space sector. This was achieved by offering the students the opportunity to develop the payload (scientific instruments or technology demonstration experiments), key satellite subsystems and the ground segment (ground stations and Mission Control) to the mission, under the coordination of ESA and SITAEL, the Industrial Prime Contractor, responsible for the satellite platform, system integration and testing, and the technical coordination of the student teams. Run by the ESA Education Office, ESEO is part of ESA Academy's Hands-on Space Programme. Ten Universities from eight ESA Member States (Estonia, Germany, Hungary, Italy, Netherlands, Poland, Spain, UK) have participated in ESEO, with more than 600 university students involved in the project since its inception. The ESEO mission will validate in-orbit the SITAEL S-50 platform (50 kg including the payload), the smallest within the SITAEL products portfolio, and hence it represents a crucial milestone of the intensive hard work in designing, developing and manufacturing innovative multi-purpose small satellites platforms.' Our Laboratory developed the Langmuir Probe Experiment (LMP) and the Power Distribution Unit (PDU) of the satellite, a rendering of the satellite is shown on Fig. 1.

'ESEO was launched aboard the Spaceflight's SSO-A: SmallSat Express dedicated rideshare mission on a SpaceX Falcon 9 launcher from the Vandenberg Air Force Base in California, (US)' in December of 2018. ESEO is situated in a
Sun-synchronous orbit with a perigee of $578.2 \mathrm{~km}$ and an apogee of $597.6 \mathrm{~km}$. The mission entered commissioning phase in March of 2019, preliminary activation of payloads was originally scheduled for the late spring or summer of the same year. However, payload activation has been put on hold since the occurrence of a communication malfunction in April. The planned nominal operational period of the satellite is six months, of which LMP will be active for one month, with the possibility of further extending it for another year [1].

\section{EFFECTS OF SOLAR ACTIVITY ON THE IONOSPHERE}

The objective of LMP is to study the plasma environment. The altitude of ESEO places it within the F-layer of the ionosphere. The ionosphere is a region of the Earth's atmosphere, where particles of atmospheric gas ionized by solar radiation form a plasma layer. The ionosphere extends from approximately $50 \mathrm{~km}$ to $1000 \mathrm{~km}$ above sea level and can be divided into layers based on electron concentration. Ionization occurs in all parts of the atmosphere that receive sunlight, but a long-term plasma layer can only form at higher altitudes, where the rate of recombination is lower due to low air pressure. The ionized layers reflect the radio waves, thus enabling long distance radio communication.

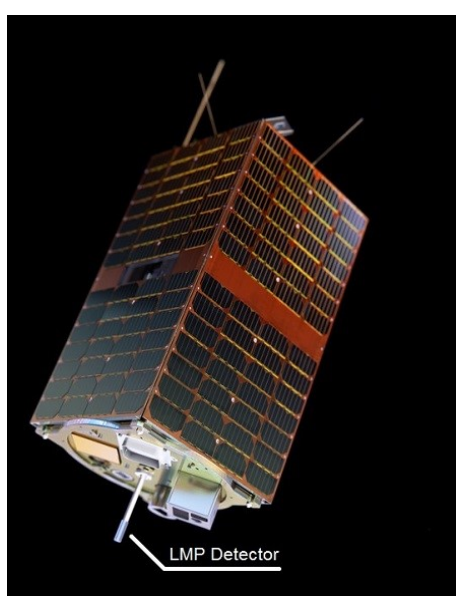

Fig. 1. The ESEO Spaceraft, credit: ESA 


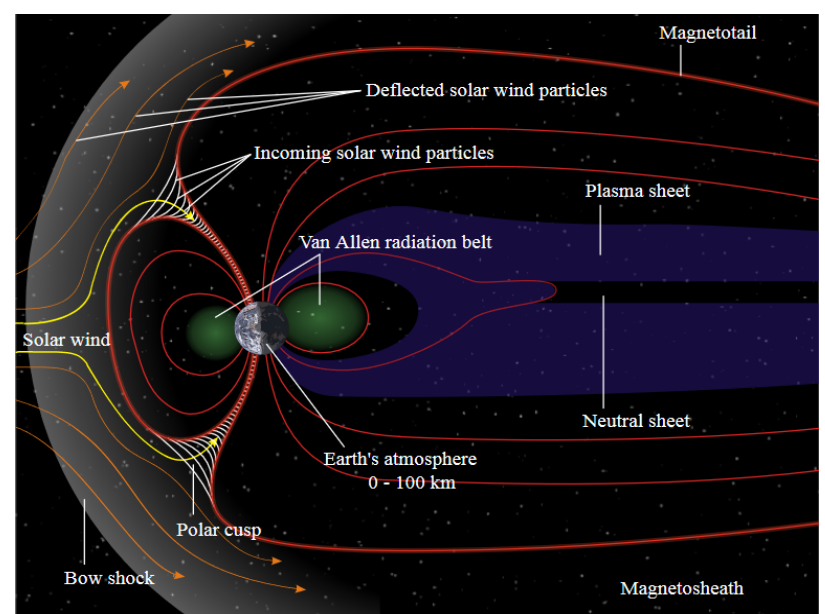

Fig. 2. Magnetosphere of the Earth, credit: William Crochot http://science.nasa.gov/newhome/headlines/guntersville98/images/mag_sketch _633.jpg

The ionosphere can be segmented into different layers depending on free electron density. The lowest layer is the D layer, above that are the $\mathrm{E}$ and $\mathrm{F}$ layers. In daylight the $\mathrm{F}$ layer breaks into the lower F1 and the upper F2 layers. At night the lower layer is neutralized through recombination. To have a better understanding of the phenomena happening in this region, we need to examine, how the Sun affects the Earth's ionosphere and magnetosphere.

The activities of the Sun can be categorized into two different groups: periodic and non-periodic activities. Periodic activities can have a period of 11 years and 27 days, 11 years is half of the period of the polarity shift of the Sun's magnetosphere, and 27 days is the length of the Sun's rotational period. Non-periodic or stochastic activities are for example the solar flares and the coronal mass ejections (CMEs). During these events low density plasma containing high-speed electrons and ions is ejected into space. These particles form the solar wind. The solar wind interacts with the Earth's magnetosphere and particles of the solar wind are added to magnetospheric plasma. The Earth's magnetic field is the field of a magnetic dipole deformed by the solar wind. The solar wind blows the magnetic field from the side facing the Sun to the opposite side of the planet, creating the magnetotail. The solar wind creates a bow shock on the side facing the Sun. At the pole cap the field lines of the Earth's magnetic field are open, this area is surrounded by the polar light belt, as shown on Fig. 2. In the ionosphere, where ESEO orbits, the Earth's magnetic field can be considered a dipole.

The solar wind has another effect on the force lines, called merging. This event occurs, when the field lines of the interplanetary magnetic field have a component in the southern direction. The magnetic field lines carried by the solar wind merge with the outer geomagnetic field lines on the side close to the Sun. During the merging the solar wind plasma is absorbed into the magnetosphere and it is directed towards the axis of the magnetotail by the electromagnetic field, building up a plasma layer in the plane of the axis of the magnetotail. Some particles of this plasma layer are accelerated into the Aurora Oval. Upon collision, they transfer energy into the local plasma, ultimately causing a temperature increase, which causes an upward drift on high and mid-high latitudes. As a result, the density of the $\mathrm{O}_{2}$ and $\mathrm{N}_{2}$ molecules will be higher compared to the density of the $\mathrm{O}$ atoms, and because of this the speed of recombination will be high enough to decrease the electron density.

CMEs and solar flares also cause disturbances in the geomagnetic field and the ionosphere. During negative ionospheric storms appear there is a large decrease in electron density. These storms are typical in mid and high latitudes. During positive ionospheric storms electron density increases, they can occur on lower latitudes. The so-called ionospheric troughs are also worth mentioning. They can appear in the lower part of the F-layer but can be detected in the orbit of ESEO in case of intense geomagnetic storms. The typical sign of these phenomena is decreased electron density [2].

\section{SCIENTIFIC OBJECTIVES}

Both spatial and temporal anomalies can be observed in electron density. At the orbit of ESEO there are three major areas of interest: the geomagnetic equatorial plane, the South-Atlantic Anomaly and geomagnetic latitudes under and over the polar light belt.

At the geomagnetic equator the product of $\mathbf{E} \times \mathbf{B}$ points upwards due to the horizontal component of the geomagnetic field. This causes an upward drift in ion density, which creates 'plasma bubbles' with decreased electron density along the geomagnetic field lines. These plasma bubbles can have a size of hundreds of kilometers and can disturb radio communication, which effect aircraft navigational systems.

The axis of the geomagnetic field is tilted away from the rotational axis of the Earth, and because of this the inner VanAllen belt dips lower into the atmosphere. This causes an anomaly in the electron density over the South Atlantic, here the flow of particles has a much higher flux, which can damage electronic equipment. Satellites in low-Earth orbit passing over this area need to be prepared for this type of hazard and because of this it is recommended to monitor this anomaly.

At the polar light belt the electron density has a maximum compared to the lower and higher geomagnetic latitudes. Therefore, from the analysis of the electron density over this area the location and the latitude of the polar light belt could be observed, which is possible due to the polar orbit of ESEO.

Some notable temporal anomalies are the daily anomaly and the winter anomaly. The daily anomaly is, when the maximum of electron density is delayed from noon to afternoon. To properly observe this anomaly the experiment should take measurements at the same place at different times, but this is not possible due to the sun-synchronous orbit of ESEO, however, comparing measurements taken at close longitudes might still be useful in studying this anomaly. Another temporal anomaly is the winter anomaly: during the winter of the northern hemisphere the measured electron density here is higher, than at the southern hemisphere during the winter of the southern hemisphere. The operation time of LMP will be only one month, and because of this we cannot observe this anomaly, however, if the mission is extended for a further year there is a possibility. 


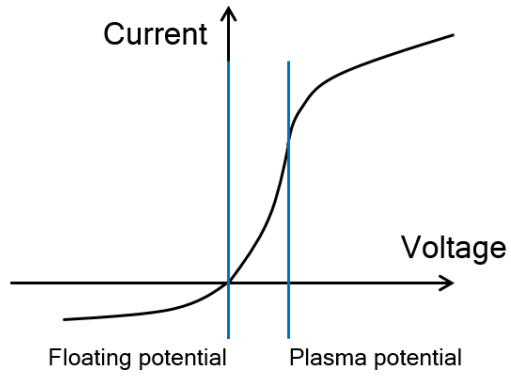

Fig. 3. UI curve of a Langmuir probe

\section{THE LANGMUIR PROBE PRINCIPLE}

\section{A. Introduction}

A Langmuir probe is a device used to determine the physical characteristics of a plasma using in-situ measurements. It works by immersing two conductors in the plasma, applying an excitation voltage between them and measuring the current flowing through one or both of the conductors. If the surface area of one of the conductors is significantly larger than that of the other the configuration is referred to as 'single pole', if not it is a 'double pole'. In single pole configurations the larger conductor can be the container wall in case of a plasma container or the chassis of a spacecraft. The electron density, electron temperature and ion temperature of the plasma can be determined by analyzing the probe current. By varying the excitation voltage, the current of the probe will also vary according to the UI curve, which is characteristic of the plasma. The shape of the curve is highly dependent on the three plasma parameters mentioned above, the typical shape is shown on Fig. 3. A measurement using a Langmuir probe consists of recording the UI curve and using curve fitting to determine the value of the plasma parameters.

\section{B. Understanding the UI curve}

The current flowing through the Langmuir probe is provided by the ions and electrons of the plasma. The probe current can be expressed as a sum of its two constituent currents the electron and ion current. The shape of the two currents is similar, electron current is positive and ion current is negative. The magnitude of the electron current is significantly higher than that of the ion current, due to the lower mass and consequently higher mobility of electrons. The plasma potential $\left(\mathrm{U}_{\mathrm{P}}\right)$ splits the voltage range into two regions. The plasma potential is the potential of the undisturbed plasma (also called bulk plasma), which is considered to be uniform in the vicinity of the probe. If the probe potential is higher than the plasma potential, the probe attracts electrons and repels ions. Similarly, at potentials lower than the plasma potential the probe attracts ions and repels electrons. Therefore, the ion and electron currents can be further subdivided into repelling and attracting (also called saturation) regions as shown on Fig. 4 [3] [4].

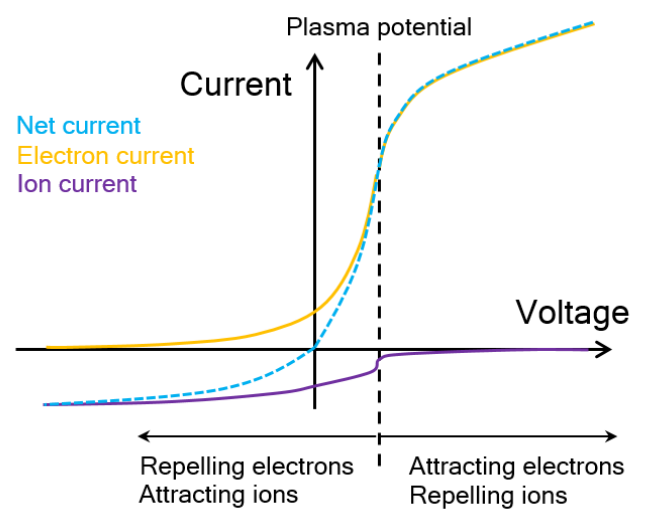

Fig. 4. Langmuir probe current decomposition

The magnitude of both the electron and ion current in their respective repelling regions is an exponential function of ratio of the probe voltage and the electron/ion temperature, as shown in (1). Where $e$ is the elementary charge, $k$ is the Boltzmann constant and $n$ is the ion or electron density, $U_{P}$ is the plasma potential and $T$ is the appropriate temperature.

$$
|I| \sim n \cdot \exp \left(e\left|U-U_{P}\right| / k T\right)
$$

This formula shows that the further the probe voltage is from the plasma potential the more repulsion the particles have to overcome and thus their current contribution decreases. Only those particles can reach the surface of the probe whose kinetic energy is greater than the energy of the electric field of the probe. The velocity of the particles follows a Maxwell-Boltzmann distribution, precise derivation of the formula is given by Shun'ko in [2]. In the saturation region all particles can reach the probe regardless of kinetic energy, however, the probe current still increases with the voltage, because the current collecting region around the probe (the sheath) grows with increasing voltage. The saturation current is proportional to the square root of the probe voltage [3] [5].

$$
|I| \sim n \cdot \sqrt{ }\left|U-U_{P}\right|
$$

The total probe current is the sum of the ion and electron currents. In the saturation regions the probe current approximately equals the respective saturation current, and thus it is possible to use these sections of the UI curve to estimate the electron and ion densities. Note, that at the so-called 'floating potential' the current is zero, this potential is in the range of ion attraction and electron repulsion. Any conductor left undisturbed in the plasma will be charged to floating potential after a sufficiently long time. Floating potential is always lower than the plasma potential. Because of the greater mobility of electrons, a slight negative charge is needed to decrease the electron current, so the currents are of equal magnitude. Since the spacecraft chassis is at floating potential, the UI curve measured by an on-board Langmuir probe will have its origin at the floating potential and the plasma potential will appear as positive. 
The region between the floating and plasma potentials is referred to as the retardation region. In the upper part of this region the electron current is dominant, the net current of the probe approximately equals the electron current and the electron temperature can be obtained. The ion current cannot be determined by this measurement, because in the ion repelling region the ion current is overshadowed by the electron saturation current.

\section{Additional considerations}

Besides the basic principle of operation, there are also some other phenomena present with Langmuir probes. The most important may be the Debye length. When a conductor is immersed in plasma the electric potential in the surrounding plasma shows an exponential decay towards the plasma potential, the Debye length is the characteristic length of this exponential decay. It is important to place the probe sufficiently far away from the satellite, so it 'sees' the undisturbed plasma. For this purpose, one Debye length away from the satellite would be enough, however, the probe has its own disturbing field, thus a distance of two Debye lengths between the probe and the satellite is a safe choice [2].

Another point to consider is the average velocity of plasma particles. In deriving the current of the Langmuir probe, we relied upon the assumption that the population of incoming particles are symmetric in velocity space, meaning there is not a single distinguished direction where the particles are coming from, rather they arrive randomly from all directions. The velocity of the electrons and ions does not reach a common equilibrium, instead they both reach a state of equilibrium amongst themselves, thus the plasma can be characterized by two velocity distributions, one for ions and one for electrons. Because of the lower mass the average velocity of electrons is much higher than that of ions, even at minimum electron temperature the average velocity is above $100000 \mathrm{~m} / \mathrm{s}$, which is much higher than the average velocity of the spacecraft (approximately $7500 \mathrm{~m} / \mathrm{s}$ ). However, the average ion velocity varies between $2800 \mathrm{~m} / \mathrm{s}$ and $8500 \mathrm{~m} / \mathrm{s}$, which is comparable to the velocity of the spacecraft, which can influence measurement results.

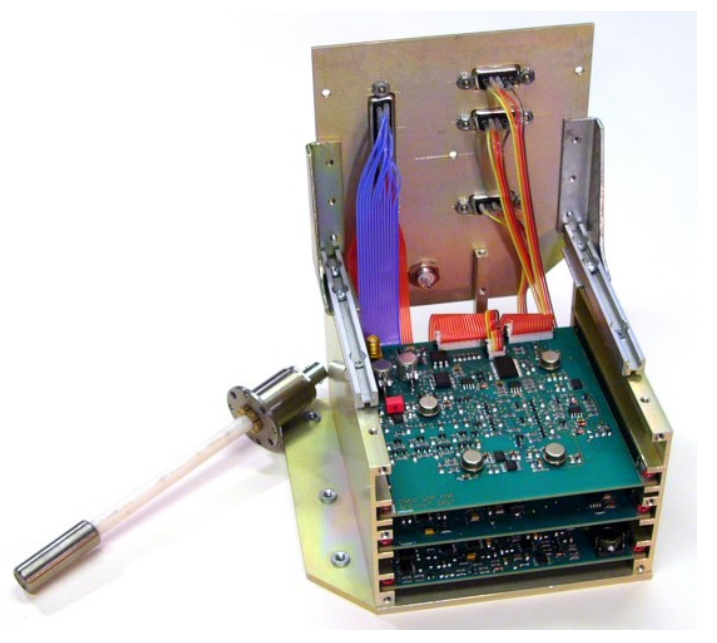

\section{DESCRIPTION OF THE LMP INSTRUMENT}

\section{A. Overview}

As we have seen, a Langmuir probe needs to record the UI curve for the plasma characteristics to be assessed. The on-board instrument needs to provide the excitation voltage, measure the current, and store the digitized results for later transmission to ground, where the analysis takes place. The instrument also needs to perform measurements automatically to achieve the desired spatial resolution. Fulfilling all these requirements necessitates a complex electronic support equipment alongside the probe. The probe and its mechanical support, collectively referred to as the Langmuir Detector (LDE) is located on the bottom of the satellite, which is normally nadir-pointing. The electronic box, called the Langmuir Control Box (LCB) is housed within the satellite and it is connected to the LDE via triaxial cable to minimize noise. The LCB contains three electronic boards: the amplifier, the on-board data handler (OBDH) and the power supply. The amplifier is responsible for producing the excitation voltage and converting the probe current into a voltage signal able to be digitized. The OBDH performs analog/digital conversions, controls the probe voltage, stores and forwards data towards the transmitter, oversees the timing of the measurements and the operational modes.

\section{B. Detector design}

LMP uses a single, cylindrical electrode $39 \mathrm{~mm}$ in length and $13 \mathrm{~mm}$ in diameter. The electrode is made of titanium to minimize the current due to photoemission [5]. It is fitted on top of a $90 \mathrm{~mm}$ long ceramic insulator rod. The length of the insulator is over twice the length of the maximal Debye length of $40 \mathrm{~mm}$. An image of the detector is shown on Fig 5. The surface area of the electrode is approximately $17 \mathrm{~cm}^{2}$. The surface area is directly proportional to the magnitude of the probe current; therefore, a large enough surface area is necessary to achieve a good signal-to-noise ratio in the current. However, the surface of the satellite needs to be significantly larger than the surface of the probe for the spacecraft potential to remain unaffected by the biasing of the probe, as explained in [5]. Mass constraints and mechanical dimensions also present an upper limit for the size of the probe.

\section{Amplifier design}

The amplifier needs to accurately set the probe voltage and produce a voltage signal proportional to the probe current. The probe bias voltage can be set anywhere within the range of $\pm 8.7 \mathrm{~V}$ with an accuracy of $17 \mathrm{mV}$. Because the probe current has a large dynamic range (it can range from picoamperes to milliamperes) the current-to-voltage amplifier uses compensating current technique with a variable feedback network to implement multiple ranges. The amplifier is capable of automatic range control, but the OBDH can overtake this function if necessary. The amplifier also has a built-in test network which can simulate different levels of probe current, it is used for precise in-flight monitoring of the gain and offset of the amplifier. A simplified block scheme of the amplifier is shown on Fig 6.

Fig. 5. The LMP instrument 


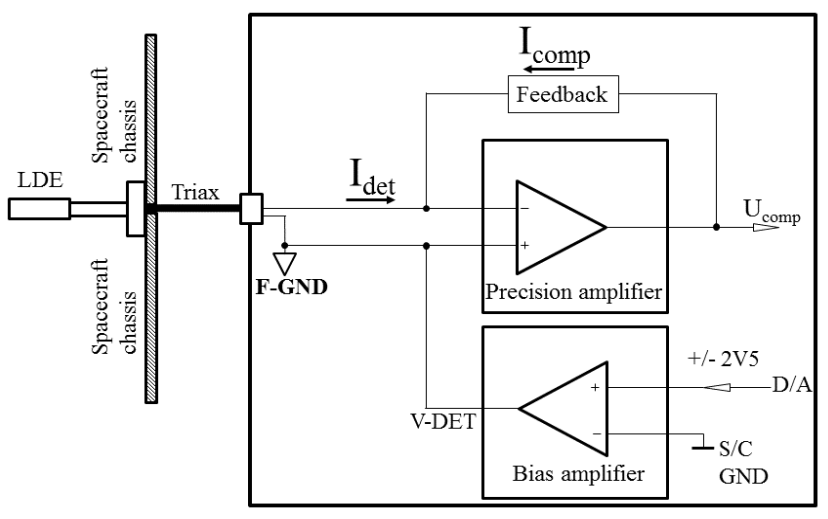

Fig. 6. Simplified block scheme of the amplifier

\section{Operational modes}

To map the entire UI curve the probe bias voltage is swept across the selected voltage range while both the probe current and voltage are recorded. The voltage sweep is performed in 100 steps, the default sweep time is $300 \mathrm{~ms}$, the probe spends $3 \mathrm{~ms}$ at each voltage level, which is sufficient time for the stepresponse of the amplifier-probe system to settle. Considering that the average velocity of the spacecraft is approximately $7500 \mathrm{~m} / \mathrm{s}$ one UI curve will be recorded over a distance of $2.25 \mathrm{~km}$. LMP alternates between four different sweep patterns shown on Fig. 7. Two of the sweeps have a section with lower voltage difference between the step in the middle, which allows for a more detailed sampling of the retardation region, where the slope is the steepest. The sweep can be performed either with increasing or decreasing voltage.
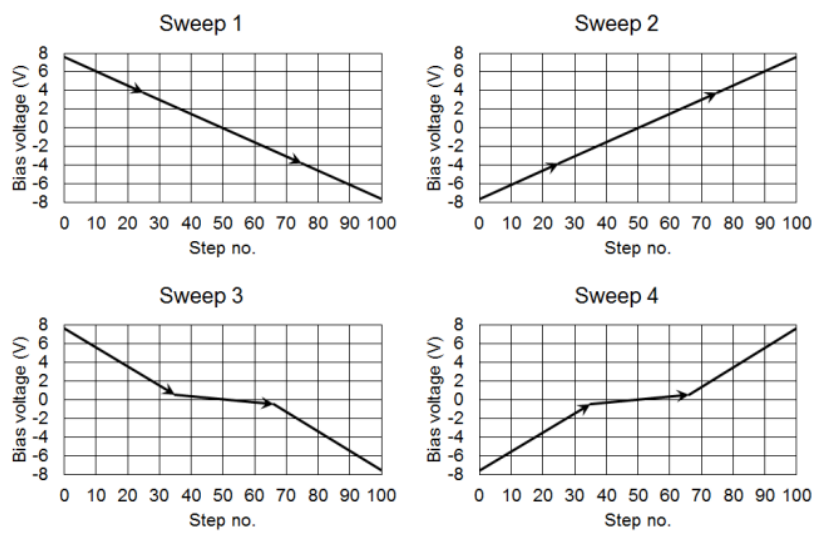

Fig. 7. Bias voltage sweep profiles
LMP can gather data in three operational modes: nominal, adaptive and self-test operation, other operational modes, such as fail-safe are not discussed here. In nominal mode a sweep is performed every second, which means that a measurement is performed every $7.75 \mathrm{~km}$ or $0.0064^{\circ}$ assuming a circular orbit. In adaptive mode a new UI curve is recorded whenever there is a larger change in electron density, which is proportional to the electron saturation current. This operational mode can generate large amounts of data and a quick consumption of memory. Entering adaptive mode can be triggered via telecommand. Sweep time, measurement frequency, voltage range and voltage step can also be configured by telecommand.

\section{CONCLUSION}

In this paper the LMP experiment was presented. LMP is an experiment designed to study the plasma environment of the upper ionosphere using the Langmuir probe principle. The measurement method was explained, and a brief description of the instrumentation was given. LMP will be able to determine the ion and electron density and electron temperature of the plasma. The scientific goal of the mission is to study the plasma anomalies, which will help to better understand the effects of the solar activity on the geomagnetic field. Currently the ESEO mission is in its commissioning phase, in-orbit verification of the payloads is yet to be performed.

\section{REFERENCES}

[1] Official website of ESEO (2019): https://www.esa.int/Education/ESEO

[2] Pál Bencze, "Bevezetés a Nap-Földfizikába” MTA CSFK Geodéziai és Geofizikai Intézet és a Nyugat-magyarországi Egyetem Kiadó, Sopron, 2014.

[3] E. V. Shun'ko, "Langmuir Probe in Theory and Practice", Universal Publishers: Boca Raton, Florida, 2009, pp. 6-17.

[4] Robert L. Merlino, "Understanding Langmuir probe current-voltage characteristics", American Journal of Physics 75, pp. 1078, 2007.

[5] J.-P. Lebreton et al., "The ISL Langmuir probe experiment processing onboard DEMETER:Scientific objectives, description and first results", Planetary and Space Science 54, pp. 472-48, 2006. 